\title{
Single-energy CT predicts uric acid stones with accuracy comparable to dual-energy CT-prospective validation of a quantitative method
}

\author{
Johan Jendeberg $^{1}$ (D) $\cdot$ Per Thunberg $^{2}$ (D) Marcin Popiolek $^{3} \cdot$ Mats Lidén $^{1}$ (ID \\ Received: 21 August 2020 / Revised: 10 December 2020 / Accepted: 21 January 2021 / Published online: 26 February 2021 \\ (C) The Author(s) 2021
}

\begin{abstract}
Objectives To prospectively validate three quantitative single-energy CT (SE-CT) methods for classifying uric acid (UA) and non-uric acid (non-UA) stones.

Methods Between September 2018 and September 2019, 116 study participants were prospectively included in the study if they had at least one 3-20-mm urinary stone on an initial urinary tract SE-CT scan. An additional dual-energy CT (DE-CT) scan was performed, limited to the stone of interest. Additionally, to include a sufficient number of UA stones, eight participants with confirmed UA stone on DE-CT were retrospectively included. The SE-CT stone features used in the prediction models were (1) maximum attenuation (maxHU) and (2) the peak point Laplacian (ppLapl) calculated at the position in the stone with maxHU. Two prediction models were previously published methods (ppLapl-maxHU and maxHU) and the third was derived from the previous results based on the k-nearest neighbors $(\mathrm{kNN})$ algorithm $(\mathrm{kNN}-\mathrm{ppLapl-maxHU})$. The three methods were evaluated on this new independent stone dataset. The reference standard was the CT vendor's DE-CT application for kidney stones.

Results Altogether 124 participants (59 \pm 14 years, 91 men) with 106 non-UA and 37 UA stones were evaluated. For classification of UA and non-UA stones, the sensitivity, specificity, and accuracy were 100\% (37/37), 97\% (103/106), and 98\% (140/ 143), respectively, for kNN-ppLapl-maxHU; 95\% (35/37), 98\% (104/106), and 97\% (139/143) for ppLapl-maxHU; and 92\% (34/37), 94\% (100/106), and 94\% (134/143) for maxHU.

Conclusion A quantitative SE-CT method (kNN-ppLapl-maxHU) can classify UA stones with accuracy comparable to DE-CT.

\section{Key Points}

- Single-energy CT is the first-line diagnostic tool for suspected renal colic.

- A single-energy CT method based on the internal urinary stone attenuation distribution can classify urinary stones into uric acid and non-uric acid stones with high accuracy.

- This immensely increases the availability of in vivo stone analysis.
\end{abstract}

Keywords Urolithiasis · Uric acid · Urinary calculi · Multidetector computed tomography

\section{Abbreviations}

AUC Area under the receiver operating characteristics curve

Johan Jendeberg

johan.jendeberg@gmail.com

1 Department of Radiology, Faculty of Medicine and Health, Örebro University, 70185 Örebro, Sweden

2 Department of Medical Physics, Faculty of Medicine and Health, Örebro University, Örebro, Sweden

3 Department of Urology, Örebro University Hospital, Örebro, Sweden
CI Confidence interval

DE-CT Dual-energy computed tomography

HU Hounsfield unit

kNN K-nearest neighbors

maxHU Maximal attenuation in a region of interest

non-UA Non-uric acid

PACS Picture archiving and communication system

ppLapl Peak point Laplacian

ROC Receiver operating characteristics

SE-CT Single-energy computed tomography

UA Uric acid 


\section{Introduction}

Urinary stone disease continues to be an increasing reason for health care admissions worldwide, with an incidence of $7 \%$ among women and $11 \%$ among men in the USA in 2010 [1]. In northern Europe, an increasing frequency of uric acid (UA) stones has been reported [2]. Approximately $7-11 \%$ of all urinary stones are UA stones [3].

Distinguishing UA from non-UA stones is of particular interest for the urologist, as the former can be treated with alkalization of the urine, allowing for secondary prophylaxis after ex vivo composition analysis of a passed or surgically removed stone [4-7]. Ideally, a UA stone is identified and dissolved in vivo, to facilitate stone passage and obviate surgical removal $[8,9]$. At present, in vivo stone composition analysis is mostly accomplished with dual-energy CT (DECT), which has shown high accuracy in several studies [10-15].

Non-enhanced single-energy CT (SE-CT) is the first-line diagnostic tool for suspected renal colic and is able to detect nearly all urinary stones with high specificity. It is highly reproducible for measuring stone size and useful for predicting spontaneous stone passage $[6,14,16]$. If, in addition, this method could also predict the composition of the stone, the patient would be able to leave the emergency room with a tailored treatment on the first day of radiologic diagnosis [9].

In a recent exploratory study on 126 urinary (22 UA and 104 non-UA) stones, Lidén correlated quantitative CT parameters in the stones to the chemical composition [17]. The highest (peak) attenuation (maxHU) of a single voxel in the stone showed to be a powerful predictor of stone composition, but, to increase the specificity, Lidén proposed a purely quantitative SE-CT method called peak point Laplacian/maxHU (ppLapl-maxHU). The ppLapl-maxHU method uses the peak attenuation in the examined stone, measured in Hounsfield units (HUs), together with the difference between this peak attenuation and the weighted mean attenuation of the surrounding voxels (Laplacian), to obtain a cutoff value of 195 $\mathrm{HU} / 1000 \mathrm{HU}$ (ppLapl/maxHU) for differentiating UA from non-UA stones. The cutoff values of the ppLapl-maxHU method resulted in high sensitivity and specificity $(95 \%$ and 99\% respectively), but with the major limitation that the cutoff values were defined post hoc, possibly causing an overestimation of the accuracy in the study. External validation on a separate dataset is therefore needed. An important advantage of the measures maxHU and ppLapl, compared to the mean attenuation often used for attenuation measurements of urinary stones, is that these two measures are point estimates, and therefore independent of segmentation parameters and reproducible.

Additional analysis of the UA- and non-UA stone data in the previous study suggested that machine learning, avoiding the sharp cutoff lines in the ppLapl-maxHU method, might perform even better than the original method [17]. K-nearest neighbors $(\mathrm{kNN})$ is a simple machine learning algorithm that uses the features of annotated cases and, through a majority vote, classifies new data according to how their neighbors in a multi-dimensional space were classified [18].

Using the same data as used in the recently developed ppLapl-maxHU method [17], a kNN algorithm was derived (kNN-ppLapl-maxHU). We hypothesized that the kNNppLapl-maxHU and the ppLapl-maxHU methods could differentiate UA from non-UA stones on previously unseen SECT with a sensitivity and specificity comparable to DE-CT and greater than using only the peak attenuation (maxHU) of the stones.

The purpose of the present study was to prospectively validate two previously published (ppLapl-maxHU and maxHU), and one derived (kNN-ppLapl-maxHU) quantitative single-energy CT methods for classifying uric acid stones on a separate, previously unseen stone dataset.

\section{Materials and methods}

This study was approved by the Regional Research Ethics Board. Written informed consent was obtained from all prospectively and retrospectively included participants.

\section{Study participants}

Between September 2018 and September 2019, 116 study participants planned for elective urinary stone $\mathrm{CT}$ examination on our DE-CT scanner, with at least one $3-20-\mathrm{mm}$ urinary stone, were prospectively included in the study to create a test dataset for validating the index tests described below (65\% of prospective, eligible participants: due to fluctuating workload, not all potentially eligible patients were asked to participate). Because of the known low prevalence of UA stones in our patient population, all eligible UA stones were included, but to keep the heterogeneity of the non-UA stones as high as possible, only one non-UA stone per participant. For inclusion and exclusion criteria, see Fig. 1.

A preliminary reading of the $\mathrm{CT}$ scans was performed in patients accepting participation. Once the radiographer had established that there was at least one urinary stone fulfilling the criteria, the patient was included and a reference DE-CT was performed. The definite decision about eligibility for inclusion was later made by a radiologist with 15 years' experience in reading abdominal CTs (J.J.).

Based on the expected low number of UA stones in our patient population, we made a pre-study-planned, additional retrospective inclusion of UA stones demonstrated on DE-CT between August 2016 and August 2018, where a SE-CT scan was available. One DE-CT stone analysis examination during 


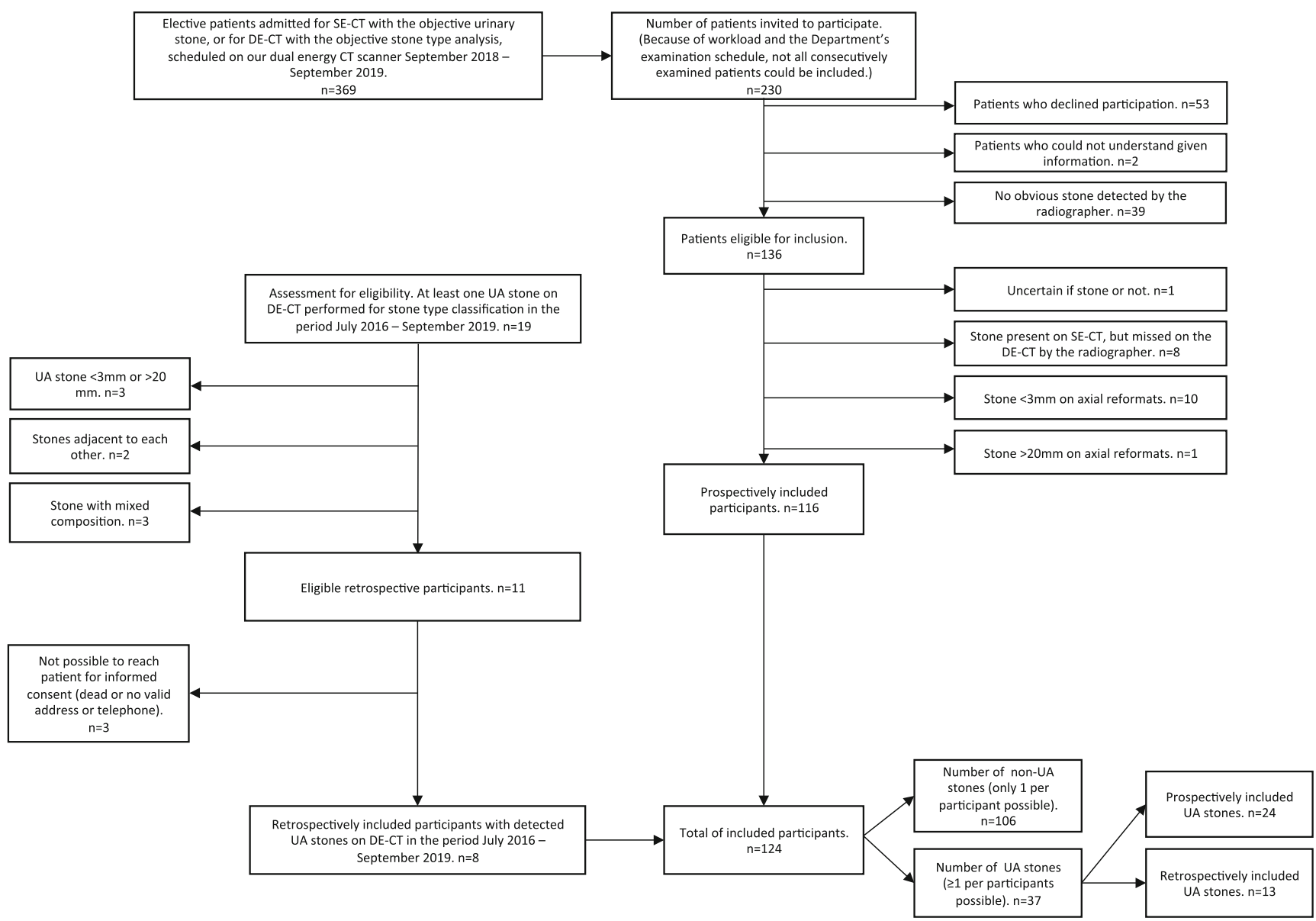

Fig. 1 Flowchart of inclusion. SE-CT, single-energy CT; DE-CT, dual-energy CT; UA, uric acid; non-UA, non uric-acid

the prospective inclusion period was missed and therefore retrospectively included. In total, eight (73\%) of the retrospectively eligible participants were included.

The sample size of $\geq 35$ UA and 100-150 non-UA stones was estimated with the objective of reaching the lower limit of a $95 \%$ confidence interval (CI) of $80 \%$, for the hypothesized sensitivity of $95 \%$, and the lower limit of $95 \%$ for the hypothesized specificity of $97 \%$.

\section{Study protocol and technical specifications}

All study participants were examined on a $2 \times 128$ channel dual-source system (Somatom Definition Flash, Siemens) using our local routine single-energy and a dual-energy protocol, for scan parameters, see Table 1 . The mean dose length product was $185 \pm 55 \mathrm{mGy} * \mathrm{~cm}$ (range 98-432) and the mean volume CT dose index was $4.5 \mathrm{mGy}$ (range 2.7-10) for the SE-CT and $189 \pm 101 \mathrm{mGy} * \mathrm{~cm}$ (range 54-637) and $16 \mathrm{mGy}$ (range 5.6-41) for the DE-CT examinations, respectively. The mean DE-CT scan length was 10.7 (range 3.8-61) cm.

The SE-CT and DE-CT reformats were pseudonymized and saved with different keys in separate folders in the local picture archiving and communication system (PACS, Sectra).

\section{Index tests}

The three index tests were created using the same data from a previous study consisting of 126 stones with known pure UA/ non-UA composition [17]. While the ppLapl-maxHU and the maxHU methods were published in the previous study, the kNN-ppLapl-maxHU method was derived for the current study. No data from the included stones in the current study was used for the development of the kNN method. None of the three index tests is commercially available at present.

\section{Index test-ppLapl-maxHU}

The two quantitative variables used for stone type prediction were (1) the highest attenuating voxel in the stone (maxHU) and (2) the value at the same position as for maxHU in a scaled Laplacian filtered image (ppLapl). An interpretation of the ppLapl is a computation of the attenuation difference between the highest attenuating voxel value and the weighted mean of the surrounding 26 voxels [17]. The pseudonymized SE-CT images were exported to MATLAB R2019a (MathWorks Inc.), where, 4 weeks after completing the inclusion process, a radiologist (J.J.) marked each included stone using 
Table 1 Scan parameters

\begin{tabular}{lll}
\hline Scan parameter & Single-energy CT (SE-CT) & Dual-energy CT (DE-CT) \\
\hline Acquisition & $128 \times 0.6$ & $32 \times 0.6$ \\
Filter & SAFIRE I30f3 & D30f \\
$\mathrm{kVp}$ & 120 & $100 / \mathrm{Sn} 140$ \\
Quality reference mAs & 70 & $210 / 162$ \\
Pitch & 1.2 & 0.7 \\
Rotation time & 0.5 & 0.5 \\
CARE-kV & Off & N/A \\
CARE dose 4D & On & On \\
Scan area & Upper kidney poles & Limited, surrounding \\
& to pelvic floor & the stone(s) \\
Field of view (mm) & 420 & 300 \\
Slice thickness/increment & $1 / 1$ & $2 / 1$ \\
$\quad($ axial reformat) (mm) & $3 / 3$ & - \\
Slice thickness/increment & & \\
$\quad$ coronal/sagittal reformat) & & \\
\hline
\end{tabular}

$S n$, tin pre-filtration; $k V$, kilovolt; $k V p$, kilovoltage peak

previously developed, semi-automatic MATLAB software to generate the maxHU and ppLapl-maxHU values (Figs. 2a and 3a). The application assigned a red color dot for UA stones and a blue dot for non-UA stones, using the cutoff values proposed in a previous study [17] (Figs. 2 and 4a).

\section{Index test-kNN-ppLapl-maxHU}

The kNN machine learning prediction method is based on the ppLapl-maxHU method. The prediction model was created in MATLAB using the nine nearest neighbors, a standard setting, and Euclidean distance. The method thereby uses the maxHU and ppLapl values of all the known stones from the previously annotated dataset, compares them with the position of an unknown stone in a scatter plot, and performs a "majority vote" between the nine stones closest to the unknown

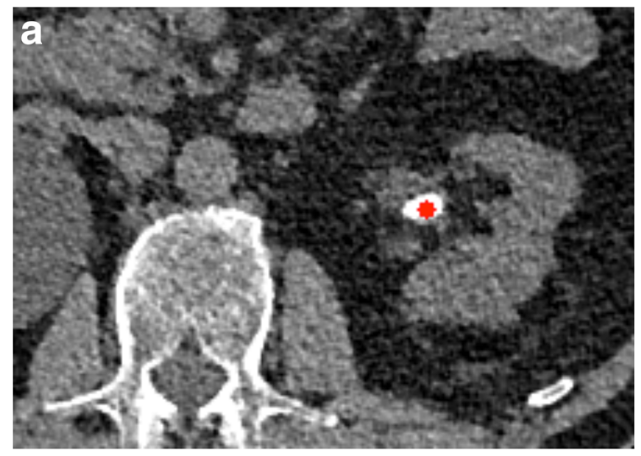

Fig. 2 Index test (a) and reference test (b) evaluating a left-sided $11 \times$ $5 \mathrm{~mm}$ uric acid (UA) kidney stone in a 66-year-old man. a Index test: 1$\mathrm{mm}$ single-energy non-enhanced axial CT scan after export to external software and marking of the kidney stone by the radiologist. The red star indicates UA composition according to the peak point Laplacianmaximum attenuation (ppLapl-maxHU) algorithm: in this case, the stone. With only two variables in the $\mathrm{kNN}$, the model can be illustrated as a curved line separating the predicted UA from non-UA stones in a scatter plot (Fig. 4b). The difference compared to the rectangular area of UA stones in the original method (Fig. 4a) is consequently the smoother curved line separating the UA from the non-UA stones.

\section{Index test-maxHU}

The third index test used only the optimal maxHU, $745 \mathrm{HU}$, defined previously [17] (Fig. 4c).

\section{Reference test/ground truth}

The stone classification (UA vs. non-UA) in DE-CT was used as ground truth for each included stone, as DE-CT

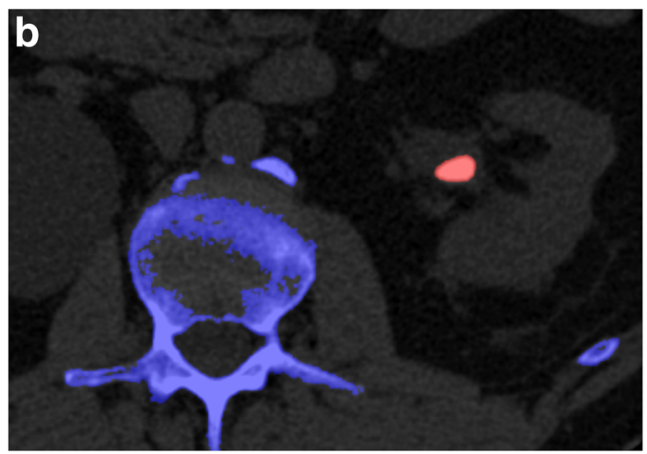

combination of a peak point attenuation of $693 \mathrm{HU}(<1000 \mathrm{HU})$ and a difference between this peak point attenuation and the mean of the surrounding 26 voxels of $98 \mathrm{HU}(<195 \mathrm{HU})$. b Reference test: Postprocessed axial dual-energy CT image using the vendor's stone composition analysis application. Red indicates stone with UA composition 


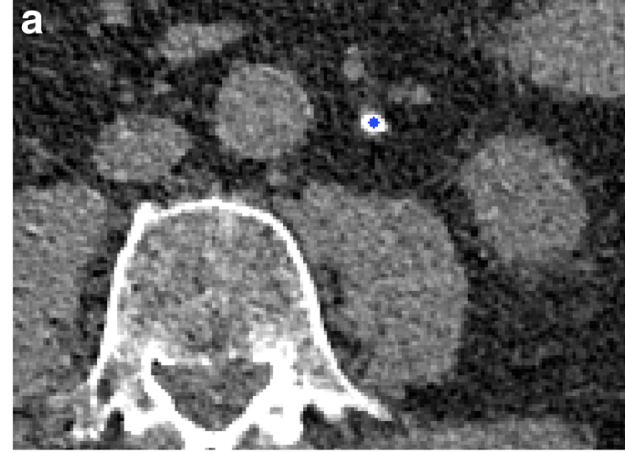

Fig. 3 Index test (a) and reference test (b) evaluating a left-sided, $5 \times$ $3 \mathrm{~mm}$ non-uric acid (non-UA) ureteral stone in a 78-year-old man. a Index test: 1-mm single-energy, non-enhanced axial CT scan after export to external software and marking of the ureteral stone by the radiologist. The blue star indicates non-UA composition, according to the peak point Laplacian-maximum attenuation (ppLapl-maxHU) algorithm: A peak

can reliably differentiate pure UA stones from non-UA stones [10-13]. The DE-CT images were analyzed, using the kidney stone application in Syngo.Via (Siemens), by a radiologist (J.J.) in conjunction with the inclusion procedure. If the stone was color-coded $>80 \%$ red (visual approximation), it was considered a UA stone, and if $>80 \%$ blue, a non-UA stone (see Figs. $2 b$ and $3 b$ ). The remaining stones $(n=7)$ were considered UA/non-UA mixed stones and removed from further analysis, since the DECT has lower reliability for mixed stones and consequently is insufficient as a reference test [19-22].

The largest diameter of each stone was measured manually in the axial plane 3-mm slice using the caliper tool in the PACS workstation with a soft window setting of C50/W400 and a zoom level of pixel-to-pixel $\times 8$.

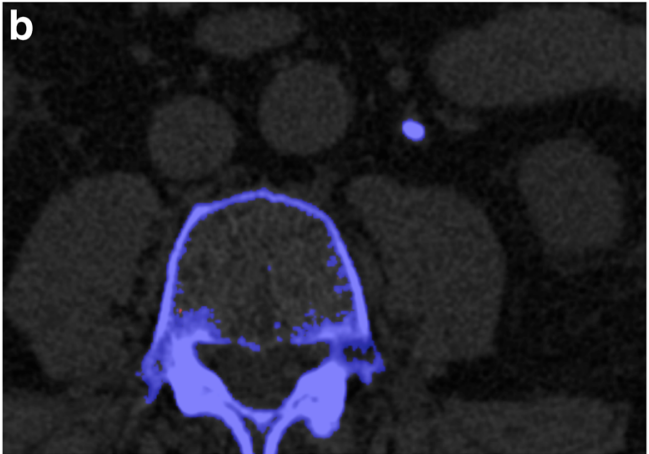

point attenuation of $\geq 1000 \mathrm{HU}$ (in this case $1398 \mathrm{HU}$ ) or a difference between this peak point attenuation and the weighted mean of the surrounding voxels of $\geq 195 \mathrm{HU}$ (in this case, $182 \mathrm{HU}$ ) defines this as a stone of non-UA composition. b Reference test: Post processed axial, dualenergy CT image using the vendor's stone composition analysis application. The blue color indicates stone with non-UA composition

\section{Statistical analysis}

Analyses were performed using IBM SPSS for Mac OS, v26.0.0.0 (SPSS Inc.), and MATLAB.

Sensitivity and specificity for, and accuracy of the prediction of UA stones, using binomial distribution and $95 \%$ CIs were calculated for the SE-CT classification methods ( $\mathrm{kNN}$ ppLapl-maxHU, ppLapl-maxHU, and maxHU).

This analysis was made primarily for all stones grouped together. Secondary analyses were performed: (1) prospectively included stones and (2) including only one UA (the most caudally positioned) or non-UA stone per patient.

Statistical significance of the difference between the index methods was tested using McNemar's test (level of significance: $p<0.05$ ).
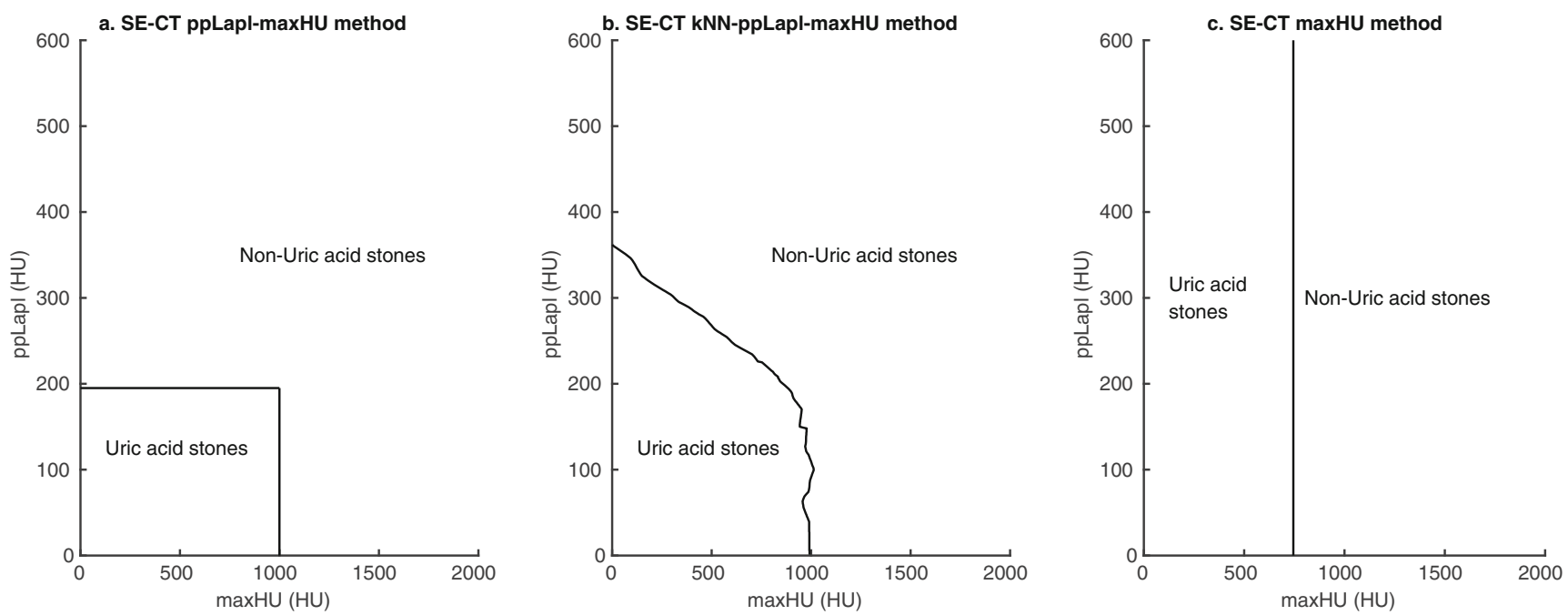

Fig. 4 Single-energy CT (SE-CT) methods for classification of uric acid (UA) stones based on maximal attenuation (maxHU) and peak point Laplacian (ppLapl). The three different SE-CT methods for the classification of UA and non-UA stones differ in the cutoffs for the radiomics

maxHU (the highest attenuating voxel in the stone) and ppLapl. a Rigid cutoffs according to the original method [14]; $\mathbf{b}$ nearest neighbor method; and $\mathbf{c}$ maxHU method 
Table 2 Stone characteristics

\begin{tabular}{|c|c|c|c|c|c|c|c|}
\hline & $\begin{array}{l}\text { All } \\
(n=143)\end{array}$ & $\begin{array}{l}\text { Non-UA } \\
(n=106)\end{array}$ & $\begin{array}{l}\mathrm{UA} \\
(n=37)\end{array}$ & $\begin{array}{l}\text { UA misclassified } \\
\text { as non-UA by } \\
\text { ppLapl-maxHU } \\
(n=2)^{* *}\end{array}$ & $\begin{array}{l}\text { Non-UA } \\
\text { misclassified } \\
\text { as UA by ppLapl- } \\
\text { maxHU }(n=2)^{* *}\end{array}$ & $\begin{array}{l}\text { Non-UA } \\
\text { misclassified } \\
\text { as UA by kNN- } \\
\text { ppLapl-maxHU } \\
(n=3)^{* *}\end{array}$ & $\begin{array}{l}\text { UA misclassified } \\
\text { as UA by kNN- } \\
\text { ppLapl-maxHU } \\
(n=0)\end{array}$ \\
\hline $\begin{array}{l}\text { Max HU (HU)* } \\
\text { ppLapl (HU)* } \\
\text { Size (mm)* }\end{array}$ & $\begin{array}{l}1092 \pm 421(223-1796) \\
240 \pm 110(47-546) \\
6.5 \pm 3.0(3.0-19)\end{array}$ & $\begin{array}{l}1286 \pm 292(536-1796) \\
282 \pm 96(51-546) \\
6.3 \pm 2.5(3.0-15)\end{array}$ & $\begin{array}{l}537 \pm 155(223-794) \\
122 \pm 39(47-212) \\
7.3 \pm 4.1(3.0-19)\end{array}$ & $\begin{array}{l}602(450-754) \\
206(201-212) \\
4.0(3.0-4.9)\end{array}$ & $\begin{array}{l}873(826-919) \\
95(79-110) \\
9.4 \pm 0.8(8.8-10)\end{array}$ & $\begin{array}{l}760(536-919) \\
133(79-209) \\
8.3(6.1-10)\end{array}$ & - \\
\hline
\end{tabular}

$S D$, standard deviation; $U A$, uric acid; $M a x H U$, maximum attenuation in a single voxel in a stone; $H U$, Hounsfield units; ppLapl-maxHU-method, peak point Laplacian-maximum attenuation, SE-CT method using the highest attenuation voxel in the stone and the weighted mean of the surrounding voxels to classify a urinary stone into UA or non-UA; $k N N$-ppLapl-maxHU-method, machine learning modification of the ppLapl-maxHU method using the nine nearest neighbors for classification

Note: *Values are mean \pm standard deviation (range). ** Standard deviation not calculated because of low number of stones

\section{Results}

Altogether 124 participants ( $59 \pm 14$ years, 91 men) with 106 non-UA and 37 UA stones were evaluated. Thirteen men had UA stones (14\%) and 78 had non-UA stones (86\%). Five women (15\%) had UA stones, whereas $28(85 \%)$ had nonUA stones. None of these stones has been used for the development of the index methods. Table 2 shows the stone characteristics. The mean age in the UA group was $69 \pm 8$ years (range 50-77) and in the non-UA group $58 \pm 14$ (21-89).

Cross-tabulations of the results of the kNN-ppLapl-maxHU, ppLapl-maxHU, and maxHU methods by the results of the reference DE-CT are shown in Table 3. Scatter plots with the distribution of maxHU and ppLapl for all stones, with cutoffs according to the three different SE-CT classification methods are shown in Fig. 5. Table 4 shows sensitivity, specificity, and accuracy for the three methods. Table 5 displays sensitivity, specificity, and accuracy of subgroups (a) prospectively included stones and (b) only one included UA stone per patient.

The area under the receiver operating characteristics (ROC) curve (AUC) for the kNN-ppLapl-maxHU, as well as the maxHU, was 0.99. The AUC cannot be computed for the ppLapl-maxHU method with two static cutoffs. Although not statistically significant $(p=0.06)$ according to the McNemar test, there was a tendency towards higher accuracy for the kNN-ppLapl-maxHU method (98\%) compared to the maxHU method (94\%). The 95\% CI for the difference was 0.5 to 9.5 percentage points.

\section{Discussion}

Single-energy CT is the first-line modality for the detection of urinary stones, whereas the in vivo stone analysis is usually conducted with dual-energy $\mathrm{CT}$, with limited availability in most emergency radiology settings [23]. The purpose of this study was to prospectively validate two previously published and one derived quantitative method (kNN-ppLapl-maxHU) for in vivo prediction of uric acid (UA) stone type using the first-line singleenergy CT scan. The kNN-ppLapl-maxHU method obtained a sensitivity for UA stones, 3-20 mm, of 100\% (37/37), a specificity of $97 \%$ (103/106), and an accuracy of $98 \%$ (140/143). The accuracy of both the ppLapl-maxHU and the maxHU method was also high, $97 \%$ and $94 \%$, respectively.

The close correlation between the attenuation and composition of a urinary stone is well known, and also the considerable overlap between UA and non-UA stones [24-26]. The $\mathrm{kNN}-\mathrm{ppLapl}-\mathrm{maxHU}$ method is purely quantitative and combines the highest attenuation within a stone with the attenuations of the surrounding 26 voxels, giving an estimate of the stone attenuation peakedness. The radiomics used in the $\mathrm{kNN}$ ppLapl-maxHU method have a logical interpretation. A small, calcium-based stone can have the same peak attenuation as a larger, UA-based stone, but the difference in attenuation between the highest attenuating voxel and the surrounding voxels is generally larger in the calcium than in the UA stone [17]. This corresponds well with previously published retrospective non-validated results. Nakada et al [24] used stone size and attenuation to analyze a sample of $17 \mathrm{UA}$ and 82 nonUA stones and reached an accuracy of 86\% (85/99); Ganesan et al [27], using stone size, attenuation, and attenuation distribution in a retrospective cohort of 52 calcium oxalate and 48 UA stones, reached an accuracy of $90 \%$; and Zhang et al [28] used stone texture analysis in a sample of $18 \mathrm{UA}$ and 32 nonUA stones and reached a sensitivity and specificity of $94 \%$.

The original methods that are prospectively validated in the present study (ppLapl-maxHU and maxHU) were developed by Lidén [17] using the distribution of the clusters of UA and non-UA stones in a scatter plot showing maxHU and ppLapl. Also, the kNN-ppLapl-maxHU algorithm in the present study was derived from only the previous data before analyzing the new data that was collected for the present study.

Consequently, a main strength of the present study compared to the previous is that the three tested algorithms were 
Table 3 Cross-tabulations of single-energy CT (SE-CT) classification of urinary stones into uric acid (UA) and non-UA stones using dual-energy CT (DE-CT) as reference. (a) kNN-peak point Laplacian-maxHU (kNN-ppLapl-maxHU); (b) peak point Laplacian-maxHU (ppLapl-maxHU); (c) maxHU

\begin{tabular}{|c|c|c|c|c|}
\hline & & \multicolumn{2}{|c|}{ Dual-energy CT } & \multirow[b]{2}{*}{ Tota } \\
\hline & & Non-UA & UA & \\
\hline \multicolumn{5}{|c|}{ a. kNN-ppLapl-maxHU * Dual-energy CT } \\
\hline \multirow[t]{3}{*}{ kNN-ppLapl-maxHU } & Non-UA & 103 & 0 & 103 \\
\hline & UA & 3 & 37 & 40 \\
\hline & Total & 106 & 37 & 143 \\
\hline \multicolumn{5}{|c|}{ Sensitivity: $100 \%$ (95\%CI 91-100\%) } \\
\hline \multicolumn{5}{|c|}{ Specificity: 97\% (95\%CI 92-99\%) } \\
\hline \multicolumn{5}{|c|}{ Accuracy: $98 \%$ (95\%CI 94-100\%) } \\
\hline \multicolumn{5}{|c|}{ b. ppLapl-maxHU * Dual-energy CT } \\
\hline \multirow[t]{3}{*}{ ppLapl-maxHU } & Non-UA & 104 & 2 & 106 \\
\hline & UA & 2 & 35 & 37 \\
\hline & Total & 106 & 37 & 143 \\
\hline \multicolumn{5}{|c|}{ Sensitivity: 95\% (95\%CI 82-99\%) } \\
\hline \multicolumn{5}{|c|}{ Specificity: $98 \%$ (95\%CI 93-100\%) } \\
\hline \multicolumn{5}{|c|}{ Accuracy: 97\% (95\%CI 93-99\%) } \\
\hline \multicolumn{5}{|c|}{ c. $\operatorname{maxHU} *$ Dual-energy CT } \\
\hline \multirow[t]{3}{*}{$\operatorname{maxHU}$} & Non-UA & 100 & 3 & 103 \\
\hline & UA & 6 & 34 & 40 \\
\hline & Total & 106 & 37 & 143 \\
\hline \multicolumn{5}{|c|}{ Sensitivity: $92 \%$ (95\%CI 78-98\%) } \\
\hline \multicolumn{5}{|c|}{ Specificity: $94 \%$ (95\%CI 88-98\%) } \\
\hline Accuracy: $94 \%(95 \% \mathrm{C}$ & & & & \\
\hline
\end{tabular}

Sensitivity and specificity for the prediction of UA stones

ppLapl-maxHU, peak point Laplacian-maximum attenuation, SE-CT method using the highest attenuation voxel in the stone and the weighted mean of the surrounding voxels to classify a urinary stone into UA or non-UA; $k N N-p p L a p l-m a x H U$, machine learning modification of the ppLapl-maxHU method using the nine nearest neighbors for classification; $\operatorname{maxHU}$, SE-CT method using the single voxel with the highest attenuation in a stone as cutoff $(<745 \rightarrow$ UA stone $)$

Dual-energy CT: Kidney stone application Syngo.Via

predefined, thereby avoiding the problem of overfitting. Although thirteen UA stones were retrospectively included, the results were similar when using only the prospectively included stones.
The kNN-ppLapl-maxHU model uses a previously acquired dataset [17] to classify a stone of unknown type through a "majority vote" between its nine closest neighbors in a scatter plot. Compared to the original method's rigid

Table 4 Sensitivity, specificity, and accuracy for the prediction of UA stones by three single-energy CT methods. Dual-energy CT as a reference standard

\begin{tabular}{llllll}
\hline & & Sensitivity, 95\% CI & Specificity, 95\% CI & Accuracy, 95\% CI \\
\hline All included stones (37 UA, 106 non-UA). & kNN-ppLapl-maxHU & $100 \%(37 / 37)$ 91-100\% & 97\% (103/106) 92-99\% & $98 \%(140 / 143) 94-100 \%$ \\
& ppLapl-maxHU & $95 \%(35 / 37) 82-99 \%$ & $98 \%(104 / 106) 93-100 \%$ & $97 \%(139 / 143) 93-99 \%$ \\
& maxHU & $92 \%(34 / 37) 78-98 \%$ & $94 \%(100 / 106) 88-98 \%$ & $94 \%(134 / 143) 88-97 \%$ \\
\hline
\end{tabular}

$U A$, uric acid; Non-UA, non-uric acid; 95\% CI, 95\% confidence intervals; ppLapl-maxHU, peak point Laplacian-maximum attenuation, single-energy CT method using the highest attenuation voxel in the stone and the weighted mean of the surrounding voxels to classify a urinary stone into UA or non$\mathrm{UA}$; $k N N$-ppLapl-maxHU, machine learning modification of the ppLapl-maxHU method using the nine nearest neighbors for classification; maxHU, single-energy CT method using the single voxel with the highest attenuation in a stone as cutoff $(<745 \rightarrow$ UA stone $)$ 
Table 5 Sensitivity, specificity, and accuracy for the prediction of UA-stones by three single-energy CT methods (a) prospectively included stones, (b) only one included UA-stone per patient. Dual-energy CT as a reference standard

\begin{tabular}{llcll}
\hline & & Sensitivity, 95\% CI & Specificity, 95\% CI & Accuracy, 95\% CI \\
\hline $\begin{array}{l}\text { a. Prospectively included stones } \\
\text { (24 UA, 106 non-UA). }\end{array}$ & kNN-ppLapl-maxHU & $100 \%(24 / 24) 86-100 \%$ & $97 \%(103 / 106) 92-99 \%$ & $98 \%(127 / 130) 93-100 \%$ \\
& ppLapl-maxHU & $92 \%(22 / 24) 73-99 \%$ & $98 \%(104 / 106) 93-100 \%$ & $97 \%(126 / 130) 92-99 \%$ \\
& maxHU & $88 \%(21 / 24) 68-97 \%$ & $94 \%(100 / 106) 88-98 \%$ & $93 \%(121 / 130) 87-97 \%$ \\
$\begin{array}{l}\text { b. Only one included UA-stone } \\
\text { per patient }\end{array}$ & kNN-ppLapl-maxHU & $100 \%(18 / 18) 82-100 \%$ & $97 \%(103 / 106) 92-99 \%$ & $98 \%(121 / 124) 93-100 \%$ \\
$(18$ UA, 106 non-UA). & ppLapl-maxHU & $94 \%(17 / 18) 73-100 \%$ & $98 \%(104 / 106) 93-100 \%$ & $98 \%(121 / 124) 93-100 \%$ \\
& maxHU & $89 \%(16 / 18) 65-99 \%$ & $94 \%(100 / 106) 88-98 \%$ & $94 \%(116 / 124) 88-97 \%$ \\
\hline
\end{tabular}

UA, uric acid; Non-UA, non-uric acid; 95\% CI = 95\% confidence intervals; ppLapl-maxHU = peak point Laplacian-maximum attenuation, singleenergy CT method using the highest attenuation voxel in the stone and the weighted mean of the surrounding voxels to classify a urinary stone into UA or non-UA; $k N N$-ppLapl-maxHU, machine learning modification of the ppLapl-maxHU method using the nine nearest neighbors for classification; maxHU, single-energy CT method using the single voxel with the highest attenuation in a stone as cutoff $(<745 \rightarrow$ UA stone)

cutoff of $195 \mathrm{HU} / 1000 \mathrm{HU}$, the proposed kNN method is appealing, as it creates a smoother curved line between the UA and non-UA stones. In our material, however, both methods had excellent accuracy (97-98\%) and either method can be used. Given this high accuracy for both methods, achieving a statistically significant difference in accuracy between them would demand a very high number of included stones, which is difficult to achieve considering the relatively low prevalence of UA stones. An advantage of the kNNppLapl-maxHU method is its plasticity; a future addition of more training examples to the algorithm is likely to further increase its accuracy. Although our hypothesis that the accuracy of the kNN-ppLapl-maxHU would be higher than the accuracy of the method using only maxHU could not be statistically proven, there was a strong tendency towards a higher accuracy $(98 \%$ vs $94 \%(p=0.06))$.
To our knowledge, this is the first prospective validation study of a SE-CT method for in vivo classification of UA and non-UA urinary stones. The results of this study are comparable to previously published results for DE-CT [11-13].

This study has limitations. Three stones in the retrospective and four in the prospective subgroup were of mixed composition (visually $20-80 \%$ UA in the DE-CT application) and were removed from the analysis. Although a recent phantom study showed promising results in demonstrating the main stone component, using a machine learning algorithm on spectral detector DE-CT [29], no previous study has shown that DE-CT can reliably classify mixed stones in vivo. A valid reference standard was consequently not available. Most studies on DE-CT classification of UA vs. non-UA stones have been performed on pure or nearly pure $(80-90 \%)$ stones $[13$,
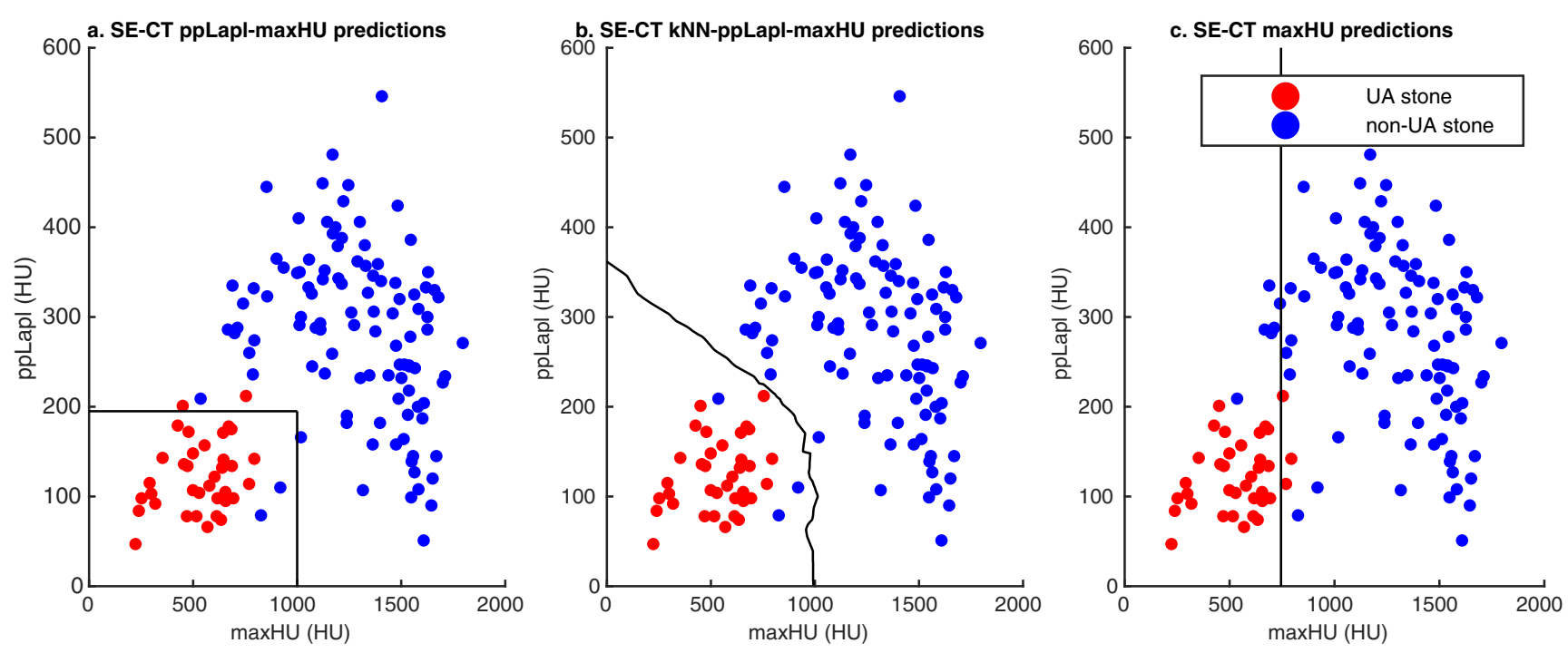

Fig. 5 Scatter plots of the three different single-energy CT (SE-CT) methods for classification of uric acid (UA) and non-UA stones. Red dots: UA stones defined by dual-energy CT (DE-CT). Blue dots: Non-
UA stones defined by DE-CT. a ppLapl-maxHU method. b kNN-ppLaplmaxHU method. c maxHU method 
19, 21, 30-33]. Consequently, the index tests are not designed for classification beyond UA/non-UA stones.

Thirteen UA stones in eight patients were included retrospectively to achieve a sufficient number of UA stones. The index tests are purely quantitative and the radiologist performing them was blinded to the results of the reference standard. The risk for bias by retrospectively including eight participants with confirmed UA stone was considered low. In addition, the sensitivity and specificity were virtually unchanged when only the prospectively included stones were analyzed; merely the CIs became broader. Because of the known low prevalence of UA stones in our population, all UA stones in a patient meeting the inclusion criteria were included, similar to previous studies [10,12, 13, 20, 28, 33-36] which may lower the heterogeneity in the UA group. In nonUA stones, the sample size could be reached with independent stones from different patients. The different inclusion strategies may thereby introduce a lower heterogeneity between UA stones, but the risk of introducing a systemic bias is considered low. When only the UA stone with the most caudal position per patient was analyzed, the sensitivity and specificity remained virtually unchanged, but with broader CIs.

The validation is made for the current settings in the CT scanner family used in this study. Validation tests on scanners from various $\mathrm{CT}$ providers need to be performed before the method can be generalized to other CT manufacturers [37]. This is an important limitation, but there is good reason to believe that similar results can be achieved after optimization, regardless of CT manufacturer, since the ppLapl-maxHU radiomics are based on the physical properties of the different stone types. Furthermore, the need for optimization of the present method depending on CT manufacturer is no different from the need for optimization depending on scanner type, of other methods, for example, a DE-CT stone type classification method.

In conclusion, this study demonstrates that a purely quantitative single-energy CT method can classify uric acid (UA) and non-UA stones in a previously unseen dataset, with accuracy comparable to dual-energy $\mathrm{CT}$, enabling immediate stone classification when a urinary stone is detected. Considering the lower cost, better availability, and lower radiation exposition, this is a promising alternative to dual-energy CT for in vivo characterization of urinary stones.

Acknowledgements Many thanks to all radiographers involved in the inclusion process and to B. Forgó for critical revision of the manuscript.

Funding Open Access funding provided by Örebro University. This study has received funding by Region Örebro Län (Nos OLL-811941 and OLL-878081) and Nyckelfonden (No OLL-787911).

\section{Compliance with ethical standards}

Guarantor The scientific guarantor of this publication is Mats Lidén.
Conflict of interest One author (ML) is the holder of a Swedish patent (SE540195C2) concerning the ppLapl method for uric acid stone prediction. The authors of this manuscript declare no relationships with any companies, whose products or services may be related to the subject matter of the article.

Statistics and biometry No complex statistical methods were necessary for this paper.

Informed consent Written informed consent was obtained from all subjects (patients) in this study.

Ethical approval Institutional Review Board approval was obtained.

\section{Methodology \\ - Prospective \\ - diagnostic or prognostic study \\ - performed at one institution}

Open Access This article is licensed under a Creative Commons Attribution 4.0 International License, which permits use, sharing, adaptation, distribution and reproduction in any medium or format, as long as you give appropriate credit to the original author(s) and the source, provide a link to the Creative Commons licence, and indicate if changes were made. The images or other third party material in this article are included in the article's Creative Commons licence, unless indicated otherwise in a credit line to the material. If material is not included in the article's Creative Commons licence and your intended use is not permitted by statutory regulation or exceeds the permitted use, you will need to obtain permission directly from the copyright holder. To view a copy of this licence, visit http://creativecommons.org/licenses/by/4.0/.

\section{References}

1. Scales CD, Smith AC, Hanley JM, Saigal CS (2012) Prevalence of kidney stones in the United States. Eur Urol 62:160-165

2. Kravdal G, Helgø D, Moe MK (2019) Kidney stone compositions and frequencies in a Norwegian population. Scand J Urol 53:139144

3. Knoll T, Schubert AB, Fahlenkamp D, Leusmann DB, WendtNordahl G, Schubert G (2011) Urolithiasis through the ages: data on more than 200,000 urinary stone analyses. J Urol 185:13041311

4. Ngo TC, Assimos DG (2007) Uric acid nephrolithiasis: recent progress and future directions. Rev Urol 9:17-27

5. Abou-Elela A (2017) Epidemiology, pathophysiology, and management of uric acid urolithiasis: a narrative review. J Adv Res 8: 513-527

6. Türk C, Petř́k A, Sarica K et al (2016) EAU guidelines on diagnosis and conservative management of urolithiasis. Eur Urol 69:468474

7. Tiselius HG, Daudon M, Thomas K, Seitz C (2017) Metabolic work-up of patients with urolithiasis: indications and diagnostic algorithm. Eur Urol Focus 3:62-71

8. Habashy D, Xia R, Ridley W, Chan L, Ridley L (2016) Impact of dual energy characterization of urinary calculus on management. J Med Imaging Radiat Oncol 60:624-631

9. Tsaturyan A, Bokova E, Bosshard P, Bonny O, Fuster DG, Roth B (2020) Oral chemolysis is an effective, non-invasive therapy for urinary stones suspected of uric acid content. Urolithiasis 48:501-507 
10. McGrath TA, Frank RA, Schieda N et al (2020) Diagnostic accuracy of dual-energy computed tomography (DECT) to differentiate uric acid from non-uric acid calculi: systematic review and metaanalysis. Eur Radiol 30:2791-2801

11. Ilyas M, Dev G, Gupta A, Bhat TA, Sharma S (2018) Dual-energy computed tomography: a reliable and established tool for In vivo differentiation of uric acid from nonuric acid renal Stones. Niger Postgrad Med J 25:52-59

12. Franken A, Alain Gevenois P, Van Muylem A, Howarth N, Keyzer C (2018) In vivo differentiation of uric acid versus non-uric acid urinary calculi with third-generation dual-source dual-energy CT at reduced radiation dose. AJR Am J Roentgenol 210:358-363

13. Nestler T, Nestler K, Neisius A et al (2019) Diagnostic accuracy of third-generation dual-source dual-energy CT: a prospective trial and protocol for clinical implementation. World J Urol 37:735-741

14. Andrabi Y, Patino M, Das CJ, Eisner B, Sahani DV, Kambadakone A (2015) Advances in CT imaging for urolithiasis. Indian J Urol 31: 185-193

15. Stolzmann P, Kozomara M, Chuck N et al (2010) In vivo identification of uric acid stones with dual-energy CT: diagnostic performance evaluation in patients. Abdom Imaging 35:629-635

16. Jendeberg J, Geijer H, Alshamari M, Cierzniak B, Lidén M (2017) Size matters: The width and location of a ureteral stone accurately predict the chance of spontaneous passage. Eur Radiol 27:47754785

17. Lidén $\mathrm{M}$ (2017) A new method for predicting uric acid composition in urinary stones using routine single-energy CT. Urolithiasis 46 : 325-332

18. Erickson BJ, Korfiatis P, Akkus Z, Kline TL (2017) Machine learning for medical imaging. Radiographics 37:505-515

19. Manglaviti G, Tresoldi S, Guerrer CS et al (2011) In vivo evaluation of the chemical composition of urinary stones using dualenergy CT. AJR Am J Roentgenol 197:W76-W83

20. Spek A, Strittmatter F, Graser A, Kufer P, Stief C, Staehler M (2016) Dual energy can accurately differentiate uric acidcontaining urinary calculi from calcium stones. World J Urol 34: 1297-1302

21. Wilhelm K, Schoenthaler M, Hein S et al (2015) Focused dualenergy CT maintains diagnostic and compositional accuracy for urolithiasis using ultralow-dose noncontrast CT. Urology 86: 1097-1103

22. Zhang GMY, Sun H, Xue H, Xiao H, Zhang XB, Jin ZY (2016) Prospective prediction of the major component of urinary stone composition with dual-source dual-energy $\mathrm{CT}$ in vivo. Clin Radiol 71:1178-1183

23. Nestler T, Haneder S, Große Hokamp N (2019) Modern imaging techniques in urinary stone disease. Curr Opin Urol 29:81-88

24. Nakada SY, Hoff DG, Attai S, Heisey D, Blankenbaker D, Pozniak M (2000) Determination of stone composition by noncontrast spiral computed tomography in the clinical setting. Urology 55:816-819
25. Spettel S, Shah P, Sekhar K, Herr A, White MD (2013) Using hounsfield unit measurement and urine parameters to predict uric acid stones. Urology 82:22-26

26. Motley G, Dalrymple N, Keesling C, Fischer J, Harmon W (2001) Hounsfield unit density in the determination of urinary stone composition. Urology 58:170-173

27. Ganesan V, De S, Shkumat N, Marchini G, Monga M (2018) Accurately diagnosing uric acid stones from conventional computerized tomography imaging: development and preliminary assessment of a pixel mapping software. J Urol 199:487-494

28. Zhang GMY, Sun H, Shi B, Xu M, Xue HD, Jin ZY (2018) Uric acid versus non-uric acid urinary stones: differentiation with single energy CT texture analysis. Clin Radiol 73:792-799

29. Große Hokamp N, Lennartz S, Salem J et al (2020) Dose independent characterization of renal stones by means of dual energy computed tomography and machine learning: an ex-vivo study. Eur Radiol 30:1397-1404

30. Grosjean R, Sauer B, Guerra RM et al (2008) Characterization of human renal stones with MDCT: advantage of dual energy and limitations due to respiratory motion. AJR Am J Roentgenol 190: 720-728

31. Cannella R, Shahait M, Furlan A et al (2019) Efficacy of singlesource rapid $\mathrm{kV}$-switching dual-energy CT for characterization of non-uric acid renal stones: a prospective ex vivo study using anthropomorphic phantom. Abdom Radiol (NY) 45:1092-1099

32. Hidas G, Eliahou R, Duvdevani M et al (2010) Determination of renal stone composition with dual-energy $\mathrm{CT}$ : in vivo analysis and comparison with x-ray diffraction. Radiology 257:394-401

33. Acharya S, Goyal A, Bhalla AS, Sharma R, Seth A, Gupta AK (2015) In vivo characterization of urinary calculi on dual-energy CT: going a step ahead with sub-differentiation of calcium stones. Acta Radiol 56:881-889

34. Apfaltrer G, Dutschke A, Baltzer PAT et al (2020) Substantial radiation dose reduction with consistent image quality using a novel low-dose stone composition protocol. World J Urol 38:2971-2979

35. Bonatti M, Lombardo F, Zamboni GA et al (2017) Renal stones composition in vivo determination: comparison between 100/ Sn $140 \mathrm{kV}$ dual-energy CT and $120 \mathrm{kV}$ single-energy CT. Urolithiasis 45:255-261

36. Zilberman DE, Ferrandino MN, Preminger GM, Paulson EK, Lipkin ME, Boll DT (2010) In vivo determination of urinary stone composition using dual energy computerized tomography with advanced post-acquisition processing. J Urol 184:2354-2359

37. Grosjean R, Daudon M, Chammas MF et al (2013) Pitfalls in urinary stone identification using $\mathrm{CT}$ attenuation values: are we getting the same information on different scanner models? Eur J Radiol 82: $1201-1206$

Publisher's note Springer Nature remains neutral with regard to jurisdictional claims in published maps and institutional affiliations. 